\title{
The Give and Take of Mental Health and Romantic Relationships
}

\author{
Christine Erickson ${ }^{1}$
}

\begin{abstract}
This paper examines the impact of mental health on romantic relationships using a symbolic interactionist approach. Specifically, this paper will focus on the dimensions of anxiety/attachment, depression, and mastery/self-esteem and how they impact romantic relationships. The impact of these dimensions is not always negative; some of the aspects can have a positive impact on relationships.
\end{abstract}

\section{Introduction}

Mental health is something that impacts various dimensions of people's lives, whether it affects them directly, a loved one or a romantic partner. Mental health is multidimensional and for this paper we will focus on the aspects of anxiety/attachment, depression, and selfesteem/mastery. The different dimensions of mental health have varying impacts. Some are more negative while others have more positive impacts, and in some cases the level of the dimension changes the impact, especially when it comes to romantic relationships (Thorsen \& Pearce-Morris, 2016). Additionally, social support and interactions can impact both mental health and relationships. When an individual struggles with their mental health it impacts a variety of areas of their life, including romantic relationships, due to the stress and the increase in dysfunction that is introduced into the relationship (Thorsen \& Pearce-Morris, 2016). For this paper I hypothesize that mental health does impact romantic relationships, I propose that it makes them more challenging but not impossible.

\section{Theory}

People are social creatures at their core. Symbolic Interactionism describes the social process of humans and the way they interact with others and their world. Symbolic interactionism suggests that humans create meanings for things within their own understanding (Blumer, 1969). One of the main ideas of symbolic interactionism is that the meanings given

\footnotetext{
${ }^{1}$ MacEwan University, Edmonton, Alberta
} 
to things are social creations that are created and defined as people interact with them (Blumer, 1969). This means that individuals need to consider the situation, their interactions, what they hope for as an outcome, as well as how their actions will impact the situation; this occurs both in the interaction between a person and the environment as well as between multiple individuals (Blumer, 1969).

Symbolic interactionism is significantly influenced by the philosopher and psychologist George Herbert Mead (Charon, 2007). Mead identified the existence of two types of social interaction, which he refers to as "conversation of gestures" and "the use of significant symbols" (Blumer, 1969, p.8). In an interaction between individuals, the individual who responds does so after taking into consideration what the other person displayed or suggested in light of what they hoped the result of the interaction would be. The responder also interprets gestures as signals of ideas, with the goal being the mutual understanding of the communication between the individuals (Blumer, 1969). In the event of a misunderstanding or confusion between individuals, the communication is affected, and the interaction would not be completed as desired (Blumer, 1969). There are three premises to symbolic interactionism, which are that "human beings act towards things based on the meanings that the things have for them" and that "meaning of such things is derived from or arises out of social interaction that one has with one's fellows" and "these meanings are handled in, and modified through an interpretive process used by the person in dealing with the things he encounters" (Blumer, 1969, p. 2). Further, Charon (2007) describes five central ideas when it comes to symbolic interactionism.

The five main ideas are "the human being must be understood as a social person, the human being must be understood as a thinking being, humans do not sense their environment directly; instead, humans define the situation they are in, the cause of human action is the result of what is occurring in our present situation, and human beings are described as active beings about their environment" (Charon, 2007, pp. 29-30). This speaks to the socialness of people at this core and is backed by the idea that being social is a continuous concept, not something that only lingers for a time, but that is present for an individuals' entire existence and directs us in how we behave as social beings (Charon, 2007). These themes depict the core of symbolic interactionism and the way it describes social interactions in our world. Interactions occur not only between individuals and the environment as well as other people, but also within themselves. This happens when interpreting information or applying meaning to an object or symbol (Charon, 2007). The interactions we have in the present and the future are partly due to the interactions we had in the past and the associations that came from those interactions that shape our understandings and processes.

In addition to this, understanding human behaviour requires consideration for social interaction through processes, situations, and the continuous flow of interactions. When looking at the interactions, it needs to be considered that the level of understanding of one's actions, the ramifications of choices, the way individuals perceive the actions they complete is all taking place in addition to what others do and how others see what they have done (Charon, 2007). The desired outcome of an interaction along with the possibility for that outcome to be achieved, as well as the anticipation of the responses to that action are also essential when attempting to understand an interaction or situation (Blumer, 1969). Another thought is that attitudes and perspectives are different and that an attitude is a response and it is an individual concept, while a perspective is a lens used to see the situation or action (Charon, 2007). Perspectives can change based on the understanding of a situation or interaction; they are varied between individuals and moments, unlike attitudes which are more consistent within an individual (Charon, 2007). A key consideration is that when it comes to people, the emphasis 
is on the interaction as opposed to personality, society, or peer influences. Even the interactions we have within ourselves result from external interactions (Charon, 2007).

When it comes to symbolic interactionism, symbols are a key piece of this theory. Symbols are social objects. They are formed socially and used intentionally in various ways and they hold shared meaning among many people who use them (Charon, 2007). Symbols are an intentional method of communication in society; they are used by individuals and are more than just physical objects that hold meaning. Language is a symbol, and it is how people communicate what they see, think, feel, or imagine. In addition, when it comes to communication, people also use unspoken gestures or cues to convey messages to others (Charon, 2007). Symbols act as a bridge between the world and how we understand it. They shift the information into a form that can be understood and used by the receiver. Being able to communicate with others allows us to learn to be social and share experiences with them and learn things like rules, values, and roles held by others or society at large (Charon, 2007). This process is how society communicates through symbols and is imperative to its function. Symbol communication is significant for cooperation among people, and without it, there would not be coordination of action or efforts (Charon, 2007).

\section{Literature Review}

Thorsen and Pearce-Morris (2016) sought to examine the long-term ramifications of adolescent mental health during the transition to adulthood, looking at the potential implications it would have on romantic relationships later in life. They looked at how mental health challenges during adolescence affected the dynamics of romantic relationships in adulthood, dynamics such as happiness or conflict within their romantic relationships. It was also understood that when a young person learns maladaptive skills it impacts them in multiple areas including romantic relationships when they are adults. Additionally, it was suggested a lack of dating experience during adolescence would have a negative outcome on future relationships as it did not allow for relationship skills to be formed (Thorsen \& Pearce-Morris, 2016). The study builds on previous research that looked at the impact of four dimensions of mental health (depressive symptoms, mastery, self-esteem, and impulsivity) on romantic relationships during the transition into adulthood (Thorsen \& Peace-Morris, 2016). The dimensions of mental health have varied impacts on relationships. Depressive symptoms may result in a lower level of happiness and an increase in conflict within the relationship. Mastery would provide a sense of strength and adaptability for individuals which would benefit their romantic relationship similarly to a higher level of self-esteem (Thorsen \& Pearce-Morris, 2016). Further, impulsivity may have a negative impact on a romantic relationship experience, because an increase in impulsivity can mean a higher chance of speaking carelessly and insensitively which results in communicative breakdown. Impulsivity can also lead to boredom in a relationship and looking for other partners, the tendency to be more aggressive and reactive, and an increase in tendency for risk taking, all of which will negatively impact a relationship (Thorsen \& Pearce-Morris, 2016).

Thorsen and Pearce-Morris (2016) hypothesized that mental health struggles as an adolescent would impact the learning and formation of the required relationship skills that lead to a successful romantic relationship as adults. They put forward three hypotheses for this study, the first being that a higher level of self-esteem and mastery is associated with a higher level of happiness and less conflict during young adulthood. Second, they predicted that when there is a higher level of depression, individuals will have fewer partners and less happiness 
and increase in conflict in relationships. Thirdly they hypothesized that increased impulsivity would lead to a higher number of partners, more conflict, and less happiness within a relationship (Thorsen \& Pearce, 2016). Their study found that there is a link between adolescent mental health and the level of happiness and conflict within a dating relationship during young adulthood (Thorsen \& Pearce-Morris, 2016). The results pointed to the presence of mastery or impulsivity during adolescence as being more likely to predict future behaviour in romantic relationships. The results also suggest that the various dimensions of mental health can have different outcomes and impact individuals both positively and negatively. For example, higher self-esteem and mastery are positive dimensions and can benefit individuals and their relationships, while depressive symptoms and impulsivity may negatively influence or complicate future romantic relationships (Thorsen \& Pearce-Morris, p. 216).

Braithwaite \& Holt-Lunstad (2017) also looked at the relationship between mental health and romantic relationships. They wanted to know which way the impact went, whether mental health affected relationships or relationships impacted mental health. In this study they found that the relationship between mental health and romantic relationships does go both ways and they each impact the other. They also found however that improving mental health did not consistently improve romantic relationships; this means that while there is a bidirectional flow of impact there is a stronger and a weaker direction (Braithwaite \& Holt-Lunstad, 2017). Further to this, they found that individuals with better mental health are more likely to be in relationships than those with mental health difficulties. It was also evident that the type of relationship needs to be considered, and that a more serious long-term relationship such as a marriage provides more benefits than a less committed relationship like cohabitation (Braithwaite \& Holt-Lunstad, 2017). Finally, they found that more significant commitments like marriage have an increased effect on the mental health of the individuals, though the mental health of the individuals does have the potential to greatly impact the quality of the relationship (Braithwaite \& Holt-Lunstad, 2017).

Braithwaite et al. (2010) looked at the impact of marriage on mental health, and they found that while marriage is beneficial to mental health it does not mean that singleness is detrimental to it. Though, it is noted that in comparison to single individuals, those in a marriage have lower levels of depression, anxiety, mood disorders and adjustment issues. The research suggests that marriage acts as a social support which in turn buffers the stressors of life and provides emotional support (Braithwaite et al., 2010). This study found that individuals who were in committed relationships generally experienced less mental health problems (Braithwaite et al., 2010).

Boysen et al., (2019) looked at mental health as a possible dealbreaker when it comes to romantic relationships. For this study they hypothesized that mental health would be a dealbreaker, and that mental illness would be considered to be more of a dealbreaker when it comes to long-term relationships than short-term relationships (Boysen et al., 2019). The results of this study suggested a varied response. Generally, though, there was support for the concept that at least some mental illnesses are potential dealbreakers. This study also looked at perceptions of mental illness as dealbreakers, and that the perception of mental illness as a dealbreaker is similar to more general relationship dealbreakers such as age or height (Boysen et al., 2019).

\section{Anxiety and Attachment}

Anxiety is a dimension of mental health that can have a negative impact on a romantic 
relationship. There are different types of anxiety, such as general anxiety and social anxiety and often anxiety will co-occur with depression (Porter \& Chambless, 2017). Studies have shown that anxiety, specifically social anxiety, is linked with relationship difficulties, and that the partners of anxious individuals could get tired of dealing with the anxiety symptoms and behaviours such as not wanting to be social due to social anxiety (Porter \& Chambless, 2017). It is also suggested that individuals with social anxiety will use relationships as refuges and be more likely to stay even if the relationship is not as successful due to the fear of the task of finding a new partner in the event of a breakup (Porter \& Chambless, 2017). Other aspects of anxiety like fear of a reaction or lack of reaction from others, fear of being a burden to others especially to a partner, doubts about self and others, and overthinking situations or interactions can impact relationships negatively (Kashdan et al., 2007). It has been shown that those with a high level of anxiety tend to have more fear surrounding relationships than those with a lower level of anxiety and are more likely to remain in relationships due to the fear of change (George et al., 2020). The anxiety about the impact they have on the other person or the way the other person will perceive them causes extra stress for them and heightens the anxiety about the relationship and whether it will last.

Individuals with anxiety often wonder if they are enough and have self-doubts. When anxious individuals express their negative emotions their self-doubt increases as they worry about the situation and the response of their partner. This causes further worries about the impacts their expressions will have on the relationship and its future (Kashdan et al., 2007). In this way anxiety acts as a barrier when it comes to vital communication within a relationship and which in turn can create more stress within the relationship and lead to a less satisfactory relationship for the other person. Anxiety can also be an issue when it comes to the potential for rejection from the partner upon disclosure of emotions and what they need from their partner within the relationship. For individuals with a lower level of anxiety it is easier to communicate and be open with their partner because the fears and anxieties about the interaction are not as dominant in their thinking. This frees them to use skills and strategies to combat the anxiety that is present as well as have energy to practice using effective communication techniques (Kashdan et al., 2007). The use of tools and skills to combat anxiety and related thinking can be a significant benefit to romantic relationships and allow for a more balanced and successful relationship that will be maintained and sustained over a longer period of time. It is healthy for a couple to express their emotions, including the negative ones and when partners listen and understand what is being said without their anxiety casting doubt, relationships and communication patterns grow stronger and partners feel understood. Expressing negative emotions is not always a problem for people with anxiety, even though the anxiety tries to make them believe it is a problem (Kashdan et al., 2007). Anxious individuals, especially those with social anxiety tend to believe that they get less support from their partners even though this is not apparent to others who observe these relationships: such a perception showcases another negative impact that anxiety has on relationships (Porter \& Chambless, 2017).

Attachment is another aspect of mental health that often goes hand in hand with anxiety and impacts the romantic relationship. Attachment influences how individuals do or do not communicate in an effort to maintain the relationship, even one that is not functioning well. Anxious individuals or those with attachment issues, will often minimize their negative emotions when communicating with their partner out of fear that the disclosure would lead to the end of the relationship (Kashdan et al., 2007). Individuals who are inappropriately attached will tend to put in more effort to sustain a negative relationship simply to avoid the end of the relationship and having to find a new one. Such individuals will look to avoid experiencing 
change; being in a relationship provides them a sense of safety and reassurance. Anxiously attached individuals will also spend more time and energy seeking reassurance and commitment from their partner, or become clingy, submissive, and overly reliant in an effort to increase the perceived closeness of the relationship they desire (Kashdan et al., 2007).

Socially anxious individuals might decide to keep their emotions and true feelings to themselves to preserve a relationship and avoid rejection, whereas a non-socially anxious individual would use the expression of emotions as a way to increase the closeness with their partner (Kashdan et al., 2007). When there is a high level of attachment anxiety present, individuals are uncertain about their partners intentions regarding their relationship. This often leads to an unsatisfactory and challenging relationship, because socially anxious people are dependent on the relationship to mediate their anxiety (George et al., 2020). Stressful interactions within a relationship such as a conflict tend to initiate the anxiety and attachment desires. In relationships where both individuals struggle with anxiety and or attachment matters can be complicated further compared to relationships where only one partner has these difficulties (George et al., 2019). Both anxiety and attachment have significant impacts on how a relationship functions as well as the way an individual perceives the relationship. Anxiety and attachment issues also influence one's core desires to remain in the relationship even if it is not as successful or fulfilling as hoped for. Further, the important role of communication in relationships is highlighted here in that it is integral for the maintenance of a healthy relationship despite the implications of anxiety and attachment to the relationship.

\section{Depression}

Depression, or depressive symptoms can have a range of impact on individuals and by extension impact romantic relationships. Not only do depressive symptoms lead to lower levels of happiness and increased conflict within relationships but they also impact next relationships due to the negative experiences of a breakup (Thorsen \& Pearce-Morris, 2016). Depression can affect an individual's behaviour and communication which can increase the chances of experiencing rejection. Behaviours such as withdrawal and isolation or anger contribute to increased stress within the relationship, as well as impacting the progression of growth in a relationship. Depression can impact social and interpersonal skills which can make relationship maintenance a struggle (Thorsen \& Pearce-Morris, 2016).

Depression or depressive symptoms impact the quality of a romantic relationship and decrease the satisfaction of the relationship. In seeking reassurance from one's partner, there is a dual aspect of togetherness but at the same time also feeling lonely (Schnapp et al., 2020; Segrin et al., 2003). The experience of loneliness while in a relationship contributes to a lower relationship quality which in turn degrades the relationship further and causes the loneliness to increase due to strain on the relationship.

Individuals with depression are more likely to speak about their relationships in a negative light even if their partner would disagree with their evaluation of the relationship (Segrin et al., 2003). It must also be said that individuals with depression can be in successful relationships and are not always experiencing loneliness or distress but that there is increased likelihood for these experiences (Segrin et al., 2003). Depression can contribute to feelings of being a burden to others and the increased need for reassurance that one's emotions have not caused strain on the relationship. Further, reassurance seeking is only a temporary solution and will come up repeatedly which can cause repeated negative emotions in the partner (Schnapp et al., 2020). In addition, the symptoms of depression or other mental health issues can lead 
individuals to question the cause of such symptoms, and if it is perceived that the present romantic relationship is the reason for the symptoms, this can lead to a change of view about the quality of the relationship. This in turn could further increase depression and or anxiety. Depression and depressive symptoms impact both relationships and the interpersonal connections of individuals who experience them (Schnapp et al., 2020). It is evident that depression can exist in a variety of intensities which can subsequently have varied impacts on individuals and their relationships.

\section{Self-Esteem and Mastery}

The impact self-esteem has on an individual varies with the level and type of selfesteem they have. Self-esteem in general affects how a person perceives a situation or interaction and when high self-esteem is present the reaction and processing of a situation is different than if the individual has a low level of self-esteem (Zeigler-Hill et al., 2011). Selfesteem impacts romantic relationships in terms of how one feels regarding the security and stability of the relationship and impacts how one would explain and describe their relationship to others. Levels of self-esteem impact the views the individual has of themselves within the relationship and in turn also impacts how they regulate their needs, emotions, doubts, and selfworth. This means that high self-esteem can be a resource for a relationship but that lower selfesteem and self-worth has the potential to increase relationship difficulties (Zeigler-Hill et al., 2011).

The level of self-esteem an individual has affects how they interpret compliments or support from others; someone with low self-esteem may not recognize or be able to accept compliments which may influence their interpretation of support that is offered by a partner can also impact the communication between partners as it affects the perceptions of interactions and responses. A person with lower self-esteem may not see interactions as positive or encouraging whereas a person with higher self-esteem might rate the same exchange as supportive and positive. The expressions of emotions and affection along with other efforts to increase intimacy are perceived and received differently based on level of self-esteem (Luerssen et al., 2017). There is a link between level of self-esteem and the tendency or likelihood to express emotion or affection toward the romantic partner and how they are understood or perceived versus how they are intended in that (Luerssen et al., 2017). Emotional support within a relationship is also associated with self-esteem and stability of the relationship; those with higher self-esteem are more likely to have a more positive experience in a relationship as they are more secure within themselves and the relationship, whereas those with lower self-esteem tend to be more anxious and self-conscious and avoidant (Knapp et al., 2016).

Low self-esteem is associated with interpersonal and relationship struggles. Those with low self-esteem are less willing to take risks within a relationship due to fear of possible rejection from the partner (Zeigler-Hill et al., 2011). Individuals with a low sense of self-esteem are less likely to enter relationships in the first place due to their feelings of low self-worth and low perception of themselves in general. (Thorsen \& Pearce-Morris, 2016). When an individual has low self-esteem any expression of affection can show vulnerability and dependence, which can feel risky. Such risks are uncomfortable due to the significant presence of self-doubt, and in an effort to protect oneself and balance the feelings of dependence, individuals may downplay the affection they receive from their partner (Luerssen et al., 2017). The simple act of expressing affection toward a partner can be perceived as a risk when an individual has low 
self-esteem due to the worry about how it will be interpreted and what it means, thus these individuals are less likely to express affection in general toward their partners. This increases the desire for individuals to exist in self-protection mode and mediate the interaction with partners when it comes to affection (Luerssen et al., 2017). Due to the fact it takes more effort and energy for individuals with low self-esteem to express and receive, these exchanges may not happen as frequently as either partner needs and this can cause challenges to the growth and longevity of the relationship. When expressing affection to their partner, individuals with low self-esteem are less likely to be fully present and invested in the moment in an effort to protect themselves in the event of a negative reaction or interaction, which again may stunt the growth and dynamics of a relationship (Luerssen et al., 2017).

When it comes to high self-esteem there are two types: secure and insecure/fragile. Each of these types are associated with different impacts on and perceptions of relationships. There is evidence to suggest that those with high self-esteem are more secure and tend to have more positive views of themselves which enables them to withstand and endure hardships more easily than those with less secure self-esteem who may not always be able to maintain positive views of themselves (Zeigler-Hill et al., 2011). Individuals with high self-esteem often react differently to situations within relationships and can better use situations as ways to increase closeness with the partner. For example, a person with high self-esteem may realize that a misunderstanding was a simple miscommunication, not take it personally, and think about a better way to communicate next time, whereas someone with low self-esteem may think the misunderstanding was their fault due to their shortcomings and be unable to move past that, thereby not finding a solution to the problem. Those with high self-esteem tend to have more positive views and attitudes about their relationships which mitigates the interactions or events that could threaten their experience (Zeigler-Hill et al., 2011).

When it comes to the types of high self-esteem, those with the insecure or fragile type may use the positive feelings from a satisfactory relationship to help them maintain their level of self-esteem - the relationship feeds their self-esteem. Individuals with high self-esteem that is secure, tend to be more realistic, anchored, and positive which increases their ability to recognize problems and their solvability thereby resisting threats to their self-esteem (ZeiglerHill et al., 2011). Secure individuals often have a solid foundational view of themselves and their worth and tend to not need constant validation from others, however those who have insecure or fragile self-esteem often have challenges when it comes to believing their worth and tend to rely more heavily on others for validation and assurance (Zeigler-Hill et al., 2011). When it comes to responding to rejection from a partner or potential partner, those with lower self-esteem tend to display negative behaviour towards their partner while those with higher self-esteem make more effort to establish closeness (Thorsen \& Pearce-Morris, 2016). Those with high self-esteem can operate with less caution, while those with low self-esteem must always be guarded, thereby limiting their ability to be part of a growing relationship. It is evident how self-esteem impacts relationships and the interactions with others in a variety of ways. Being open to communicating and exchanging expressions of affection will increase the chances of a fulfilling and long-lasting relationship, which is directly impacted by level of selfesteem (Luerssen et al., 2017).

Similar to high self-esteem, mastery can act as a resource for individuals when it comes to relationships. Mastery is the sense of agency and a person's belief in their ability to influence their environment, which helps them achieve their goals (Thorsen \& Pearce-Morris, 2016). Having a greater level of mastery increases the likelihood an individual will face or confront issues that come up in their relationship, as well as encourage and support when necessary, as opposed to when there is a lower level of mastery or agency and issues will be reacted to with 
withdrawal, denial, and/or minimization. Mastery supports a more positive reaction to stressors and allows room for skill building and ingenuity to take place which minimizes negative reactions and creates space for problems to be solved cooperatively. (Thorsen \& Pearce-Morris, 2016).

\section{Conclusion}

In conclusion, it is evident that the dimensions of mental health of anxiety/attachment, depression, and self-esteem/mastery all have varied impacts on the success and fulfillment of romantic relationships. It is also clear how the interactionism perspective applies to mental health and romantic relationships in that the interactions within relationships, how perceptions and understandings along with level of communication all contribute to a fulfilling healthy relationship. When it comes to the dimensions of mental health specifically, anxiety/attachment and depression have mostly negative impacts on romantic relationships as they have potential to have significant impacts on the individual and their abilities to communicate and manage interpersonal situations, in addition to the impact these dimensions have on the partner's perception of the behaviours (Porter \& Chambless, 2017). Depression can cause behaviours such as withdrawal, isolation, and anger and strain communication which contributes to a higher level of stress in the relationship. This increases the likelihood of rejection which in turn leads to increased difficulty maintaining the relationship (Thorsen \& Pearce-Morris, 1016). Finally, the impacts of high self-esteem and mastery, are positives when it comes to relationships and increase the likelihood of a satisfactory relationship, while low self-esteem can strain relationships when individuals hold back or second guess themselves while needing more reassurance from their partner (Thorsen \& Pearce-Morris, 2016; Zeigler-Hill et al., 2011). These findings together show support for the hypothesis that mental health impacts romantic relationships. 


\section{References}

Beeney, J. E., Stepps, S. D., Ringwald, W. R., Wright, A. G. C., Mattia, A. A., Ayers, H. E., Gebresselassie, S. H., Hallquist, M. N., Lazarus, S. A., Scott, L. N., Pilkonis, P. A. (2019). Attachment Styles, Social Behaviour, and Personality Functioning in Romantic Relationships. Personality Disorders: Theory, Research, and Treatment, 10(3), 275-285. https://dx.doi.org/10/1037/per0000317

Blumer, H. (1969). Symbolic Interactionism Perspective and Method. University of California Press.

Braithwaite, S. R., Delevi, R., \& Fincham, F. D. (2010). Romantic relationships and the physical and mental health of college students. Journal of the International Association for Relationship Research, 17(1), 1-12. https://doi.org/10.1111/j.14756811.2010.01248.x

Braithwaite, S., \& Holt-Lunstad, J. (2017). Romantic relationships and mental health. Current Opinions in Psychology, 13, 120-125. https://doi.org/10.1016/j.copsyc.2016.04.001

Charon, J. M. (2007). Symbolic Interactionism: An Introduction, An Interpretation, An Integration. Pearson Prentice Hill.

George, T., Hart, J., \& Rholes, W. S. (2020). Remaining in unhappy relationships: The roles of attachment anxiety and fear of change. Journal of Social and Personal Relationships, 37(5), 1626-1633. https://doi.org/10.1177/0265407520904156

Kashdan, T. B., Volkmann, J. R., Breen, W. E., \& Han, S. (2007). Social anxiety and romantic relationships: The costs and benefits of negative emotion expression are context-dependent. Journal of Anxiety Disorders, 21(4), 475-492. https://doi.org/10.1016/j.janxdis.2006.08.007

Knapp, D. J., Durtschi, J. A., Clifford, C. E., Kimmes, J. G., Barros-Gomes, P., \& Sandberg, J. (2016). Self-esteem and caregiving in romantic relationships: Self- and partner perceptions. Personal Relationships, 23(1), 111-123. https://doi.org/10.1111/pere.12114

Luerssen, A., Jhita, G. J., \& Ayduk, O. (2017). Putting Yourself on the Line: Self-Esteem and Expressing Affection in Romantic Relationships. Personality and Social Psychology Bulletin, 43(7), 940-956. https://doi.org/10.1177/0146167217702374

Porter, E., \& Chambless, D. L. (2017). Social Anxiety and Social Support in Romantic Relationships. Behaviour Therapy, 48(3), 335-348. https://doi.org/10.1016/j.beth.2016.12.002

Schnapp, J. E., O'Neal, D. N., \& Vaughn, A. A. (2020). Dyadic perceptions of depression and anxiety in romantic relationships. Journal of Social and Personal Relationships, 37(2), 432-449. https://doi.org/10.1177/0265407519867418

Segrin, C., Powell, H. L., Givertz, M., \& Brackin, A. (2003). Symptoms of depression, relational quality, and loneliness in dating relationships. Personal Relationships, 10(1), 25-36.

Thorsen, M. L., \& Pearce-Morris, J. (2016). Adolescent Mental Health and Dating in Young Adulthood. Society and Mental Health, 6(3), 223-245. https://doi.org/10.1177/2156869316665884

Zeigler-Hill, V., Fulton, J. J., \& McLemore, C. (2011). The role of unstable self-esteem in the appraisal of romantic relationships. Personality and Individual Differences, 51(1), 5156. https://doi.org/10.1016/j.paid.2011.03.009 